\title{
Water Vapor Transport in Carbon Nanotube Membranes and Application in Breathable and Protective Fabrics
}

\author{
Francesco Fornasiero $^{a}$ \\ a Physical and Life Sciences, Lawrence Livermore National Laboratory, 7000 East Avenue, \\ Livermore, CA 94550, USA; E-mail: fornasiero1@Ilnl.gov
}

\begin{abstract}
Membranes that selectively block or permeate water vapor find application in dehydration of gaseous streams, pervaporation, humidity control in buildings, protection of moisture sensitive electronics, and in breathable fabrics for environmental protective clothing. Many of these application areas could benefit from the incorporation of carbon nanotubes into membrane materials. Recent studies have demonstrated the ability of carbon nanotubes to enable ultrafast water vapor diffusion through their inner volume, as well as to promote dehumidification of gas streams flowing through their interstitial spaces. Membranes with porosity solely made of the inner channels of carbon nanotubes are especially promising for the development of next generation protective garments because enable a long-sought-after combination of outstanding breathability with protection.
\end{abstract}

\section{Introduction}

A wide spectrum of important applications requires membranes that selectively block or permeate water vapor. For example, the removal of water vapor from gaseous streams is critical in industrial processes such as dehydration of natural and flue gases [1], drying of compressed air $[2,3]$, and for harvesting water vapor from the atmosphere. Humidity regulation is important for air conditioning in buildings [4] and repositories for the conservation of art and paper artifacts. Moisture barriers are used for food storage [5] and protection of moisture sensitive electronics such as solar cells and organic light emitting diodes [6]. Breathable membranes, i.e. membranes that permit rapid water vapor permeation, find application in biofuel separation by pervaporation [3] and in water desalination [7] by membrane distillation [8]. Waterproof, but water-vapor-permeable membranes are widely used in the textile industry for weather protective clothing [9-11]. 
These membranes are designed to allow body-generated moisture to pass through while preventing wind and rain from penetrating the fabric, thus keeping the body dry and warm. Their selective permeability for water vapor is also interesting in medical applications, e.g., for personal cooling garments [12,13], and as components of protective clothing for first responders, military, and medical personnel $[10,14]$.

In many of the listed applications, boosting membrane permeability is very desirable. For example, in flue gas dehydration [1], membrane distillation, and pervaporation processes, a higher membrane flux can result in energy saving and/or decreased capital costs by reducing plant footprint. For apparel use, higher membrane breathability correlates strongly with increased comfort $[11,15]$. Unfortunately, performances of conventional polymeric membranes are typically limited by a trade-off between flux and selectivity [16]. Recent advances in nanotechnology have enabled the fabrication of novel membranes incorporating nanomaterials that could potentially overcome this shortcoming of polymer films [17]. Among these, carbon nanotubes (CNT) are emerging as exciting building blocks for fabrication of water-vapor selective membranes for several reasons. First and most importantly, the graphitic walls of CNTs are molecularly smooth and slippery [18••], and this property has been linked to CNTs unique ability to sustain fluid transport rates that are several orders of magnitude faster than typically found in other pores of similar sizes [19,20]. Second, while the outer surface of vertically aligned CNT forests is superhydrophobic $[21,22]$, the inner surface of CNT nanopores can be readily wetted by liquid water [23•]. Thus, water (vapor) transport can be either facilitated or hindered by judicious choices of the CNT membrane structure, permeation pathways, and operating conditions. Third, the diameter of CNTs can be synthetically tuned from sub-nanometer to tens of nanometers, and their length can be controlled on the sub-micron to millimeter scale, thus enabling a unique combination of ultrahigh aspect-ratio with small pore opening. Finally, welldefined sites for chemical functionalization at the entrance of an open CNT nanochannel can be easily targeted to create selective gates for molecular transport [24]. This localized functionalization may enable boosting membrane selectivity without a dramatic flux loss.

In this paper, we review studies published in the last five years that have highlighted the CNT potential to either enhance or block water vapor permeation through membranes by exploiting CNT geometry, wetting, and fast flow properties. We describe different types of CNT 
membranes that have been fabricated, and their specific use in applications involving water vapor transport. Special attention is given to the field of breathable and protective fabrics.

\section{CNT membrane types}

CNT membranes with a variety of structures have been fabricated and investigated for their potential use in a wide class of applications involving selective transport of water vapor. These include membranes with vertically-aligned CNTs (VACNT) working as only through pores in an otherwise impermeable film (type 1 - Figure 1) [25••]; matrix-less membranes made of ultralong, vertically-aligned CNT arrays, in which the flow pathways are the interstices among CNTs (type 2) [26••]; and membranes where CNTs are immobilized in the pores of a polymer film or partially/fully embedded into a polymer matrix (type 3) [27-30]. The permeation behavior of water vapor observed in these membrane types differs both qualitatively and quantitatively depending on the preferential transport pathways (Figure 2).

\section{Water vapor transport through CNT inner channels}

The first quantification of water vapor transport rates inside CNT nanochannels under a concentration gradient appeared only recently. Bui et al. [25] prepared type-1 CNT membranes by filling the interstitial spaces between sub-5 nm, vertically-aligned, singlewalled nanotubes (SWNTs) with parylene-N. To measure the membrane breathability, a purely diffusive steady-state transport of water vapor was established by exposing each membrane side to a nitrogen gas stream with a different relative humidity $(\mathrm{RH})$. Because water fluxes measured with a constant concentration gradient were independent of the inlet $\mathrm{RH}$ in the range of $55 \%-85 \%$, water did not condense inside the pores and transported through the CNTs as vapor. Measured water vapor diffusivity in SWNTs was 24 times larger than Knudsen diffusion predictions and comparable to bulk diffusivities $\left(\approx 0.16 \mathrm{~cm}^{2} \mathrm{~s}^{-1}\right.$ in these SWNTs vs $0.26 \mathrm{~cm}^{2} \mathrm{~s}^{-1}$ in the bulk) (Figure 3a). The magnitude of this flow enhancement $E$ with respect to Knudsen theory is in excellent agreement with that of pressure-driven nitrogen flow through these $(E=50)$ and other type-1 membranes reported in the literature [31--35]. In analogy with pressure-driven transport of pure gases, water vapor enhanced flow is likely due to the inherent wall smoothness of wellgraphitized CNT pores [36•--39]. 


\section{Water vapor transport through CNT interstitial spaces}

A very different water-vapor permeation behavior was found when the interstitial spaces

between nanotubes rather than the CNT inner channels were used as gas flow pathways [26,40] . Matrix-less membranes made of closed, 4-mm long, vertically aligned, multi-walled nanotubes (MWNTs) impeded water vapor transport by a combination of capillary condensation, reverse capillary flow, and water rejection/removal at the superhydrophobic MWNT tips [41]. This mechanism of water rejection (shown in Figure 2.B) was supported by the more efficient dehumidification at higher $\mathrm{RH}$ of the incoming gas stream [26], at lower temperature of the MWNT membrane [40], and with membranes having a reduced intertube distance ( $R_{1}$ and $R_{\mathrm{c}}$ in Figure 2) [40,41]. Thanks to a large porosity $(98.5 \%)$ and a large average distance between the MWNTs $(\sim 73 \mathrm{~nm})$, the achieved permeability of dry gases $\left(2.1 \times 19^{-9}-3.8 \times 10^{-8} \mathrm{~mol} \mathrm{~m} / \mathrm{m}^{2} \mathrm{~s} \mathrm{~Pa}\right)$ was orders of magnitude greater than the permeability of polymeric membranes typically used for water vapor separation [26]. Selectivity (defined as permeability ratio) for several polar and non-polar gases with respect to water vapor was as high as $\sim 2 \times 10^{5}$ (Figure 4 ).

\section{Water vapor transport through CNT-polymer composites}

Membranes incorporating randomly-oriented, 30-nm wide MWNTs into super-absorbing polymers were evaluated for their ability to harvest water vapor from the atmosphere [30] (type 3 - Figure 1). In these composite materials, molecular transport occurred through the polymer matrix, the CNT channels, and possibly through (sub-)nanogaps at the interface between nanotubes and polymer [42]. Addition of MWNTs increased water vapor removal from wet air by up to $45 \%$, and this improved performance was attributed to the nanotube slippery walls and to changes in the polymer-water interaction in the presence of MWNTs.

Functionalized CNTs were also immobilized in the pores of hydrophobic membranes (polytetrafluoroethyelene, polypropylene) for water desalination by membrane distillation [27-29]. CNT incorporation doubled the water vapor flux and increased salt rejection. The benefit of CNT addition was attributed to the creation of additional pathways for water vapor transport (at the tube outer surface and through the CNT inner volume) and to the increased hydrophobicity of the composite membrane that prevented salty water penetration. 
Note that, because the multiple gas permeation pathways in type-3 membranes cannot be decoupled easily, the relative importance of each transport mode to the performance improvement could not be quantified. Nevertheless, since the magnitude of flow enhancement in CNTs typically decays with increasing nanotube diameter [36] and defect density, the water vapor permeation rate through the core of the large diameter, oxidized MWNTs of these studies may be significantly lower than through pristine SWNTs [25].

\section{Application in breathable and protective fabrics}

The ability of small-diameter SWNTs to transport rapidly water vapor by diffusion has important implications for the fabrication of protective garments. To minimize physiological burden and prevent the risk of heat stress, a protective ensemble must provide a moisture vapor transport rate (MVTR) $>1500-2000 \mathrm{gr} \mathrm{m}^{-2}$ day $^{-1}$ [43], i.e. large enough to allow efficient evaporative cooling of the body through perspiration $[9,44]$. Unfortunately, achieving both high protection and high breathability in a single material is challenging, and current protective materials sacrifice breathability in order to prevent exposure to harmful agents $[44,45 \cdot]$.

Sub- $5 \mathrm{~nm}$ SWNT membranes with pore densities of about $5 \times 10^{11}$ tube $/ \mathrm{cm}^{2}$ were demonstrated to exhibit outstanding moisture vapor transport rates approaching $8000 \mathrm{gr}$

$\mathrm{m}^{-2}$ day $^{-1}$ [25], i.e. 4-fold larger than the breathability target. Notably, these transport rates were comparable or larger than those of conventional breathable fabrics tested at the same conditions (Figure 3b). In addition, the small diameter of the SWNT pores enabled complete rejection of $\geq 5$-nm analytes by size exclusion and, thus, provided protection from biological threats [25]. These results showed that, by combining high breathability and protection in a single functional material, CNT pores can effectively overcome the breathability/protection trade-off predicted by conventional diffusion theories (Figure 3a).

\section{Future outlook}

The proof-of-concept demonstration [25] of combined bio-protection and outstanding breathability in VACNT membranes has highlighted the potential of CNT nanomaterials for next-generation breathable and protective fabrics. Further development, however, is 
required to meet the demand of high comfort, increased protection from a wider spectrum of threats, mechanical robustness for incorporation in garments, and scalability of the fabrication process.

Boosting CNT membrane selectivity is expected to provide the most benefit. VACNTs with the narrowest diameters produced so far [46-48] typically contain a population of sub-nanometer CNTs, but also a tail of larger size nanotubes that extends up to $\sim 2-n m$. Thus, membranes made of these state-of-art, narrow VACNTs could easily block the permeation of hazardous species as small as 2-nm by size exclusion. Contrary to biological threats, chemical threats are small enough to permeate even through the narrowest diameter VACNT arrays achieved so far. Fortunately, the entrance of an open CNT nanochannel offers well-defined sites for chemical functionalization that can be easily targeted to create selective gates for molecular transport. Integration of chemicalthreat-responsive molecules [49•] at the CNT entrance could greatly improve the membrane protection properties toward chemical hazards.

While membranes with sub-5nm SWNTs at densities of $5 \times 10^{11}$ tube $/ \mathrm{cm}^{2}$ display a small resistance to water vapor transport, the overall fabric permeability may be detrimentally reduced if additional layers are coated on the CNT membrane surface or smaller diameter SWNTs are adopted for increased protection. To counteract this potential loss of breathability, it is desirable to increase CNT pore density. Growth of VACNT arrays with $>10^{13}$ tube $/ \mathrm{cm}^{2}$ was demonstrated in the literature [46], and their successful integration in flexible films could dramatically enhance the intrinsic CNT membrane breathability. A unique advantage of the VACNT membrane structure is that transport and mechanical properties are largely decoupled. The former are dictated by the CNT pores, the latter by the impermeable material filling the interstitial gaps between nanotubes. Thus, the filling matrix can be potentially chosen with the sole aim of optimizing mechanical properties such as flexibility, mechanical robustness, tear resistance, etc. Incorporation of barrier polymers typically used in protective garments would be especially beneficial. Scaling-up fabrication of VACNT membranes remains challenging. VACNT membranes best exploit the transport properties of the CNT inner pores, but so-far have been demonstrated only at $\mathrm{cm}^{2}$ scale with a batch process that starts from CVD-grown VACNT forests $[32,34,50-53]$. Solution based methods have the potential to produce large area 
VACNTs and could be integrated in roll-to-roll processes. These methods aim to align solution-dispersed CNTs with an external field and lock the nanomaterial orientation by in-situ polymerization [54]. In spite of considerable progress [55], this fabrication route has not demonstrated yet adequate packing densities of SWNT pores across the membrane thickness to form breathable films.

\section{Conclusions}

Among the recently developed types of CNT membranes, those using VACNTs as a building block best exploit the unique nanomaterial wetting and transport properties and have enabled permeation rates that greatly surpass those of competing polymer membranes. The unique geometry and hydrophobicity of matrix-less CNT membranes could offer a low-energy, cost-effective strategy to control humidity in industrial application. The recent demonstration that water-vapor transport driven by a relative humidity gradient is exceptionally fast inside SWNTs has revealed the potential of these nanomaterials for use in breathable and protective fabrics. These quantitative measurements of water vapor diffusion in CNTs have also provided key missing information to design membranes for pervaporation and membrane distillation. Widespread adoption of VACNT membranes by the industry requires resolving challenges related to their large-scale manufacturability at a competitive cost, as well as further enhancing membrane selectivity.

\section{Acknowledgments}

This work was supported by the Chemical and Biological Technologies Department of the Defense Threat Reduction Agency (DTRA-CB) via grant BA12PHM123 in the "Dynamic Multifunctional Materials for a Second Skin D[MS ${ }^{2 "}$ "program. Work at LLNL was performed under the auspices of the US Department of Energy under contract DE-AC52-07NA27344. 


\section{References}

1. Sijbesma H, Nymeijer K, van Marwijk R, Heijboer R, Potreck J, Wessling M: Flue gas dehydration using polymer membranes. Journal of Membrane Science 2008, 313:263-276.

2. Baker RW: Gas Separation. In Membrane Technology and Applications. Edited by: John Wiley \& Sons, Ltd; 2012:325-378.

3. Baker RW: Pervaporation. In Membrane Technology and Applications. Edited by: John Wiley \& Sons, Ltd; 2012:379-416.

4. Zhang LZ: Progress on heat and moisture recovery with membranes: From fundamentals to engineering applications. Energy Conversion and Management 2012, 63:173-195.

5. Powers TH, Calvo WJ: 9 - Moisture regulation A2 - Ahvenainen, Raija. In Novel Food Packaging Techniques. Edited by: Woodhead Publishing; 2003:172-185.

6. Kawano K, Pacios R, Poplavskyy D, Nelson J, Bradley DDC, Durrant JR: Degradation of organic solar cells due to air exposure. Solar Energy Materials and Solar Cells 2006, 90:3520-3530.

7. Baker RW: Other Membrane Processes. In Membrane Technology and Applications. Edited by: John Wiley \& Sons, Ltd; 2012:521-557.

8. Curcio E, Drioli E: Membrane distillation and related operations - A review. Separation and Purification Reviews 2005, 34:35-86.

9. Lomax GR: Breathable polyurethane membranes for textile and related industries. Journal of Materials Chemistry 2007, 17:2775-2784.

10. Gugliuzza A, Drioli E: A review on membrane engineering for innovation in wearable fabrics and protective textiles. Journal of Membrane Science 2013, 446:350-375.

11. Mukhopadhyay A, Midha VK: 2 - Waterproof breathable fabrics. In Handbook of Technical Textiles (Second Edition). Edited by: Woodhead Publishing; 2016:27-55.

12. Rothmaier M, Weder M, Meyer-Heim A, Kesselring J: Design and performance of personal cooling garments based on three-layer laminates. Medical \& Biological Engineering \& Computing 2008, 46:825-832.

13. Davis SL, Wilson TE, White AT, Frohman EM: Thermoregulation in multiple sclerosis. Journal of Applied Physiology 2010, 109:1531-1537.

14. Schreuder-Gibson HL, Truong Q, Walker JE, Owens JR, Wander JD, Jones WE: Chemical and biological protection and detection in fabrics for protective clothing. MRS Bulletin 2003, 28:574-578.

15. Mukhopadhyay A, Midha VK: A Review on Designing the Waterproof Breathable Fabrics Part I: Fundamental Principles and Designing Aspects of Breathable Fabrics. Journal of Industrial Textiles 2008, 37:225-262.

16. Freeman BD: Basis of permeability/selectivity tradeoff relations in polymeric gas separation membranes. Macromolecules 1999, 32:375-380.

17. Kim W-g, Nair S: Membranes from nanoporous 1D and 2D materials: A review of opportunities, developments, and challenges. Chemical Engineering Science 2013, 104:908-924.

18. Secchi E, Marbach S, Niguès A, Stein D, Siria A, Bocquet L: Massive radius-dependent flow slippage in carbon nanotubes. Nature 2016, 537:210-213.

-• This recent paper reports the first measurement of pressure-driven water flow rates through individual carbon nanotubes with diameters as small as $30-\mathrm{nm}$, and reveals unexpectedly large, 
radius-dependent surface slippage. On the contrary, no slippage is found in boron nitride nanotubes that are crystallographically similar to carbon nanotubes, but electronically different. This contrast highlights the unique fluidic properties of carbon nanotube nanochannels.

19. Holt JK: Carbon Nanotubes and Nanofluidic Transport. Advanced Materials 2009, 21:19.

20. Guo S, Meshot ER, Kuykendall T, Cabrini S, Fornasiero F: Nanofluidic Transport through Isolated Carbon Nanotube Channels: Advances, Controversies, and Challenges. Advanced Materials 2015, 28:5871-5877.

21. Zhang L, Resasco DE: Single-Walled Carbon Nanotube Pillars: A Superhydrophobic Surface. Langmuir 2009, 25:4792-4798.

22. Lee CH, Johnson N, Drelich J, Yap YK: The performance of superhydrophobic and superoleophilic carbon nanotube meshes in water-oil filtration. Carbon 2011, 49:669-676.

23. Pascal TA, Goddard WA, Jung Y: Entropy and the driving force for the filling of carbon nanotubes with water. Proceedings of the National Academy of Sciences of the United States of America 2011, 108:11794-11798.

- This molecular dynamics study quantifies the entropy, enthalpy, and free energy of water confined in carbon nanotubes with diameters in the range of $0.8-2.7 \mathrm{~nm}$. For all sizes, water inside a CNT is more stable than in the bulk, but the nature (entropic versus enthalpic) of the favorable confinement of water changes dramatically with CNT diameter. These simulation results explain and further support the counterintuitive experimental observation of spontaneous water filling in CNT channels.

24. Noy A, Park HG, Fornasiero F, Holt JK, Grigoropoulos CP, Bakajin O: Nanofluidics in carbon nanotubes. Nano Today 2007, 2:22-29.

25. Bui N, Meshot ER, Kim S, Peña J, Gibson PW, Wu KJ, Fornasiero F: Ultrabreathable and Protective Membranes with Sub-5 nm Carbon Nanotube Pores. Advanced Materials 2016, 28:6020-6020.

-• This manuscript shows that flexible membranes with $<5-\mathrm{nm}$ CNT pores exhibit outstanding moisture vapor transport rates and completely reject $>5$-nm analytes and bio-threats by size exclusion. To the author knowledge, this is the first study demonstrating that, when a concentration gradient is used as driving force, $\mathrm{CNT}$ nanochannels can sustain vapor transport rates exceeding Knudsen diffusion theory by more than one order of magnitude.

26. Yoon D, Lee C, Yun J, Jeon W, Cha BJ, Baik S: Enhanced Condensation, Agglomeration, and Rejection of Water Vapor by Superhydrophobic Aligned Multiwalled Carbon Nanotube Membranes. Acs Nano 2012, 6:5980-5987.

•- In thi experimental work, the authors demonstrate efficient dehydration of wet light gases permeating through the intestices of aligned multiwalled carbon nanotubes. A new mechanism of dehydration is proposed that combines condensation, agglomeration, and rejection of water vapor by superhydrophobic CNT arrays.

27. Bhadra M, Roy S, Mitra S: A Bilayered Structure Comprised of Functionalized Carbon Nanotubes for Desalination by Membrane Distillation. Acs Applied Materials \& Interfaces 2016, 8:19507-19513. 
28. Bhadra M, Roy S, Mitra S: Flux enhancement in direct contact membrane distillation by implementing carbon nanotube immobilized PTFE membrane. Separation and Purification Technology 2016, 161:136-143.

29. Gethard K, Sae-Khow O, Mitra S: Water Desalination Using Carbon-NanotubeEnhanced Membrane Distillation. ACS Applied Materials \& Interfaces 2011, 3:110114.

30. Roy S, Hussain CM, Mitra S: Carbon nanotube-immobilized super-absorbent membrane for harvesting water from the atmosphere. Environmental Science-Water Research \& Technology 2015, 1:753-760.

31. Majumder M, Chopra N, Hinds BJ: Mass Transport through Carbon Nanotube Membranes in Three Different Regimes: Ionic Diffusion and Gas and Liquid Flow. Acs Nano 2011, 5:3867-3877.

- This paper studies ionic diffusion and pressure-driven gaseous and liquid flow through the core of CNT pores. Consistently with previous reports, the magnitude of pressure-driven flow for both gases and liquids is orders of magnitude larger than Knudsen and "no-slip" hydrodynamic flow predictions, respectively. On the contrary, diffusive transport of ions of different charge and size is close to bulk diffusion expectations. By comparing as produced CNTs with tip- and corefunctionalized CNTs, this work gives a direct experimental confirmation that the dramatically enhanced flow in CNTs is related to the large slip at the wall of pristine CNTs.

32. Holt JK, Park HG, Wang YM, Stadermann M, Artyukhin AB, Grigoropoulos CP, Noy A, Bakajin O: Fast mass transport through sub-2-nanometer carbon nanotubes. Science 2006, 312:1034-1037.

33. Zhang L, Zhao B, Wang X, Liang Y, Qiu H, Zheng G, Yang J: Gas transport in verticallyaligned carbon nanotube/parylene composite membranes. Carbon 2014, 66:11-17.

34. Hinds BJ, Chopra N, Rantell T, Andrews R, Gavalas V, Bachas LG: Aligned multiwalled carbon nanotube membranes. Science 2004, 303:62-65.

35. Kim S, Fornasiero F, Park HG, Bin In J, Meshot E, Giraldo G, Stadermann M, Fireman M, Shan J, Grigoropoulos CP, et al.: Fabrication of flexible, aligned carbon nanotube/polymer composite membranes by in-situ polymerization. Journal of Membrane Science 2014, 460:91-98.

36. Skoulidas AI, Ackerman DM, Johnson JK, Sholl DS: Rapid transport of gases in carbon nanotubes. Physical Review Letters 2002, 89:4.

•• This is the first simulation study predicting that transport diffusivities of light gases in carbon nanotubes are orders of magnitude larger than in any known microporous material. The exceptionally high transport rates in nanotubes are shown to be a result of the inherent smoothness of the nanotube walls.

37. Chen HB, Johnson JK, Sholl DS: Transport diffusion of gases is rapid in flexible carbon nanotubes. Journal of Physical Chemistry B 2006, 110:1971-1975.

38. Sokhan VP, Nicholson D, Quirke N: Transport properties of nitrogen in single walled carbon nanotubes. Journal of Chemical Physics 2004, 120:3855-3863.

39. Ackerman DM, Skoulidas AI, Sholl DS, Johnson JK: Diffusivities of Ar and Ne in carbon nanotubes. Molecular Simulation 2003, 29:677-684.

40. Jeon W, Yun J, Khan FA, Baik S: Enhanced water vapor separation by temperaturecontrolled aligned-multiwalled carbon nanotube membranes. Nanoscale 2015, 7:14316-14323. 
41. Jongju Y, Wonjae J, Khan FA, Jinkee L, Seunghyun B: Reverse capillary flow of condensed water through aligned multiwalled carbon nanotubes. Nanotechnology 2015, 26:235701 (235710 pp.)-235701 (235710 pp.).

42. Roy S, Ntim SA, Mitra S, Sirkar KK: Facile fabrication of superior nanofiltration membranes from interfacially polymerized CNT-polymer composites. Journal of Membrane Science 2011, 375:81-87.

43. Lu X, Nguyen V, Zhou M, Zeng X, Jin J, Elliott BJ, Gin DL: Crosslinked Bicontinuous Cubic Lyotropic Liquid-Crystal/Butyl-Rubber Composites: Highly Selective, Breathable Barrier Materials for Chemical Agent Protection. Advanced Materials 2006, 18:3294-3298.

44. Wartell MA, Kleinman MT, Huey BM, Duffy LM: Strategies to Protect the Health of Deployed U.S. Forces: Force Protection and Decontamination. Washington, D.C.: National Academy Press; 1999.

45. A. Brewer S: Recent Advances in Breathable Barrier Membranes for Individual Protective Equipment. Recent Patents on Materials Science 2011, 4:1-14.

- This review covers patents that have been published recently on breathble materials that can be incorporated into garments to provide protection from toxic chemicals.

46. Zhong G, Warner JH, Fouquet M, Robertson AW, Chen B, Robertson J: Growth of Ultrahigh Density Single-Walled Carbon Nanotube Forests by Improved Catalyst Design. Acs Nano 2012, 6:2893-2903.

47. Chen G, Seki Y, Kimura H, Sakurai S, Yumura M, Hata K, Futaba DN: Diameter control of single-walled carbon nanotube forests from 1.3-3.0 $\mathrm{nm}$ by arc plasma deposition. Scientific Reports 2014, 4:3804.

48. Cui K, Kumamoto A, Xiang R, An H, Wang B, Inoue T, Chiashi S, Ikuhara Y, Maruyama S: Synthesis of subnanometer-diameter vertically aligned single-walled carbon nanotubes with copper-anchored cobalt catalysts. Nanoscale 2016, 8:1608-1617.

49. Belger C, Weis JG, Egap E, Swager TM: Colorimetric Stimuli-Responsive Hydrogel Polymers for the Detection of Nerve Agent Surrogates. Macromolecules 2015, 48:7990-7994.

- In this paper, the authors developed copolymers that exhibit a strong colorimetric response to chemical warfare agents and a simultaneous volume collapse upon contact with chemical threats. Both colorimetric and mechanical responses are reversible, and the polymer original state can be regenerated by exposing the material to a base. When covalently attached to the entrance of CNT pores in a breathable CNT membrane, polymers of this kind could enable dynamic protection from chemical warfare agents.

50. Majumder M, Chopra N, Andrews R, Hinds BJ: Nanoscale hydrodynamics - Enhanced flow in carbon nanotubes. Nature 2005, 438:44-44.

51. Yu M, Funke HH, Falconer JL, Noble RD: High Density, Vertically-Aligned Carbon Nanotube Membranes. Nano Letters 2009, 9:225-229.

52. Du F, Qu LT, Xia ZH, Feng LF, Dai LM: Membranes of Vertically Aligned Superlong Carbon Nanotubes. Langmuir 2011, 27:8437-8443.

53. Pilgrim GA, Leadbetter JW, Qiu F, Siitonen AJ, Pilgrim SM, Krauss TD: Electron Conductive and Proton Permeable Vertically Aligned Carbon Nanotube Membranes. Nano Letters 2014, 14:1728-1733. 
54. Mauter MS, Elimelech M, Osuji CO: Nanocomposites of Vertically Aligned Single-Walled Carbon Nanotubes by Magnetic Alignment and Polymerization of a Lyotropic Precursor. Acs Nano 2010, 4:6651-6658.

55. Castellano RJ, Akin C, Giraldo G, Kim S, Fornasiero F, Shan JW: Electrokinetics of scalable, electric-field-assisted fabrication of vertically aligned carbonnanotube/polymer composites. Journal of Applied Physics 2015, 117:214306-214306.

\section{Figure Captions}

Figure 1. Fabrication process for three types of CNT membranes. Type 1: VACNT membranes that transport fluids only through the inner volume of the CNT pores. Type 2: Matrix-less VACNT membranes, where the transport pathways are the interstitial spaces between CNTs. Type 3: Mixed matrix membranes, in which one or both CNT ends are covered by a permeable polymer. A-C: SEM images of the cross-section (A-B) and surface (C) of the three types of membranes. Reproduced with permission from [25] (type 1) - Copyright (C) 2016, John Wiley and Sons; [26] (type 2) - Copyright (C) 2012, American Chemical Society; and [29] (type 3) Copyright (C) 2011, American Chemical Society.

Figure 2. Mechanism of water vapor transport through (A) the endohedral CNT volume and (B) the interstitial space in-between VACNTs. A: Water diffuses through the CNT channels as vapor and with rates that greatly exceed Knudsen predictions [25]. B: According to Kelvin equation, water vapor condenses in the gaps between VACNTs. Formation of an asymmetric liquid meniscus originates a large Laplace pressure difference in the fluid that drives liquid water backwards [41]. $Q_{K n}$ is the volumetric flow rate for Knudsen diffusion; $Q$ the measured volumetric flow rate; $M$ the molecular weigth; $V_{\mathrm{m}}$ the molar volume; $R_{c}$ the capillary radius; $T$ the absolute temperature; $R$ the universal gas constant; $E$ the enhancment factor; $\mu$ is the fluid viscosity; $L$ the pore length; $L_{s}$ the slip length at the pore wall; $R_{1}$ and $R_{2}\left(=R_{c} / \cos \theta\right)$ the radii of curvature of the two menisci; $P_{\text {sat }}^{K}$ and $P_{\text {sat }}$ the saturation pressure of water vapor in equilibrium with a curved liquid meniscus and next to a flat interface, respectively; $\gamma$ the surface tension; $\theta$ the contact angle. 
Figure 3. Water vapor transport through VACNT membranes, commercial breathable fabrics, and other porous membranes. A: Single-pore water vapor diffusivity. The pore size dependences predicted by bulk, transition, and Knudsen diffusion equations are represented by the black dotdashed line, solid black line, and the blue dashed line, respectively. Points represent experimental results for several porous membranes. Inset: Enhancement factor defined as ratio of measured to predicted diffusivity. $\mathrm{PC}=$ polycarbonate; $\mathrm{AAO}=$ anodic aluminum oxide. Upper $\mathrm{x}-$ axis: Typical dimensions (nm) of biological threats. B: MVTR (bars), corresponding total ( $R_{\text {tot }}$, circles) and boundary layer resistance ( $R_{B L}$, dashed line) to water vapor transport for CNT membranes and several commercial breathable fabrics based on macroporous films (ePTFE, eVent, NoeShell) or dense polymer laminates (Gore-Tex Pro Shell, Sympatex). Reproduced with permission from [25] - Copyright (C) 2016, John Wiley and Sons.

Figure 4. Gas transport through matrixless VACNT membranes. A: Gas selectivity with respect to water vapor when the inlet gas is at $100 \%$ RH. B: Permeability comparison. Data for matrixless VACNT membranes are shown as solid squares, while control data from the literature are represented by the open symbols. Red circles = molecular sieves; green diamonds = carbon nanotubes (inner volume); blue stars = polymeric membranes for water vapor separation. Reproduced with permission from [26] - Copyright (C) 2012, American Chemical Society. 
Type 1

VACNT membrane
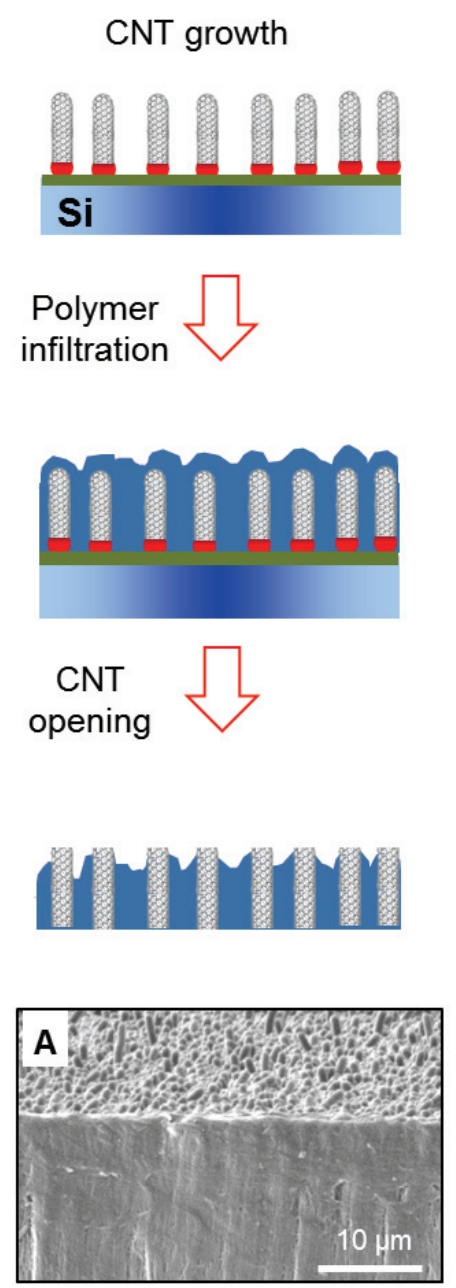

Type 2

Matrix-less VACNT membrane
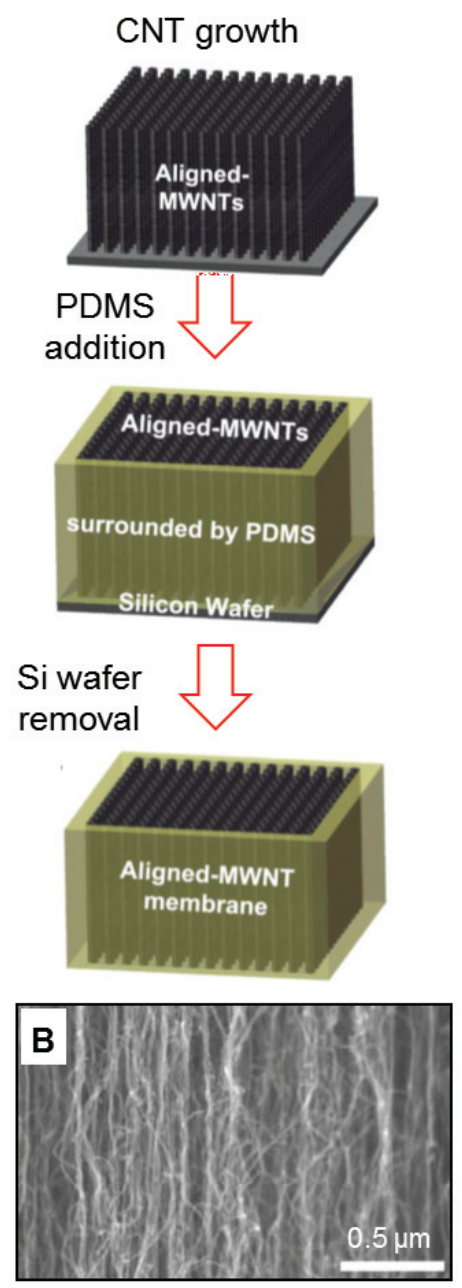

Type 3

Mixed matrix membrane

Mixing/Sonication
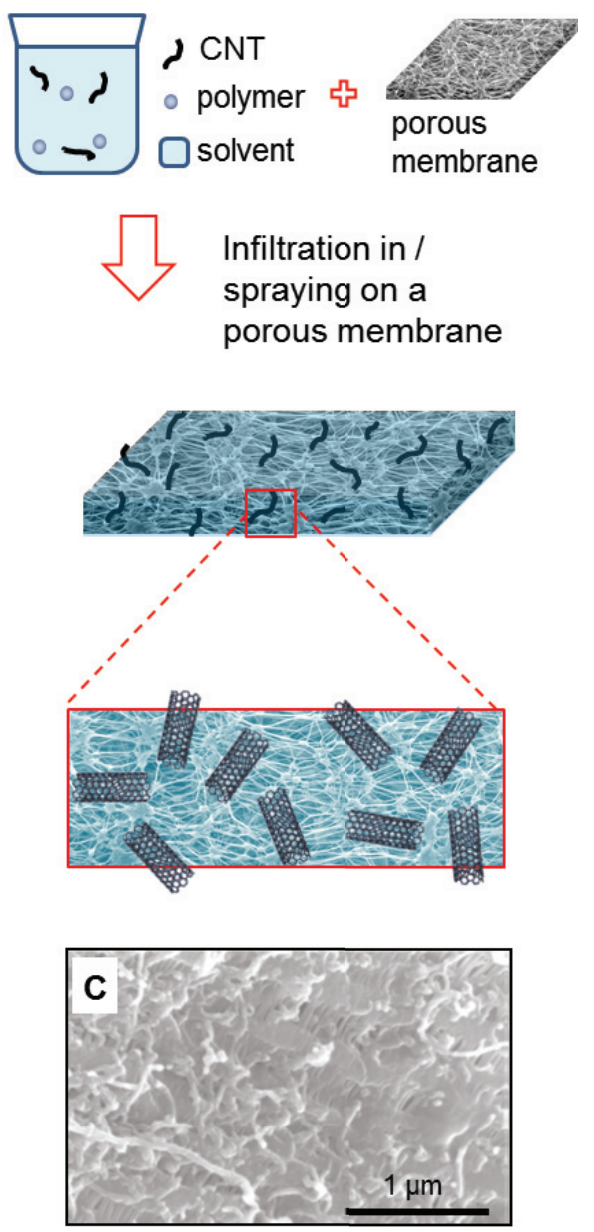

Figure 1. Fabrication process for three types of CNT membranes. Type 1: VACNT membranes that transport fluids only through the inner volume of the CNT pores. Type 2: Matrix-less VACNT membranes, where the transport pathways are the interstitial spaces between CNTs. Type 3: Mixed matrix membranes, in which one or both CNT ends are covered by a permeable polymer. A-C: SEM images of the cross-section (A-B) and surface (C) of the three types of membranes. Reproduced with permission from [25] (type 1) - Copyright (C) 2016, John Wiley and Sons; [26] (type 2) - Copyright (C) 2012, American Chemical Society; and [29] (type 3) Copyright (C) 2011, American Chemical Society. 


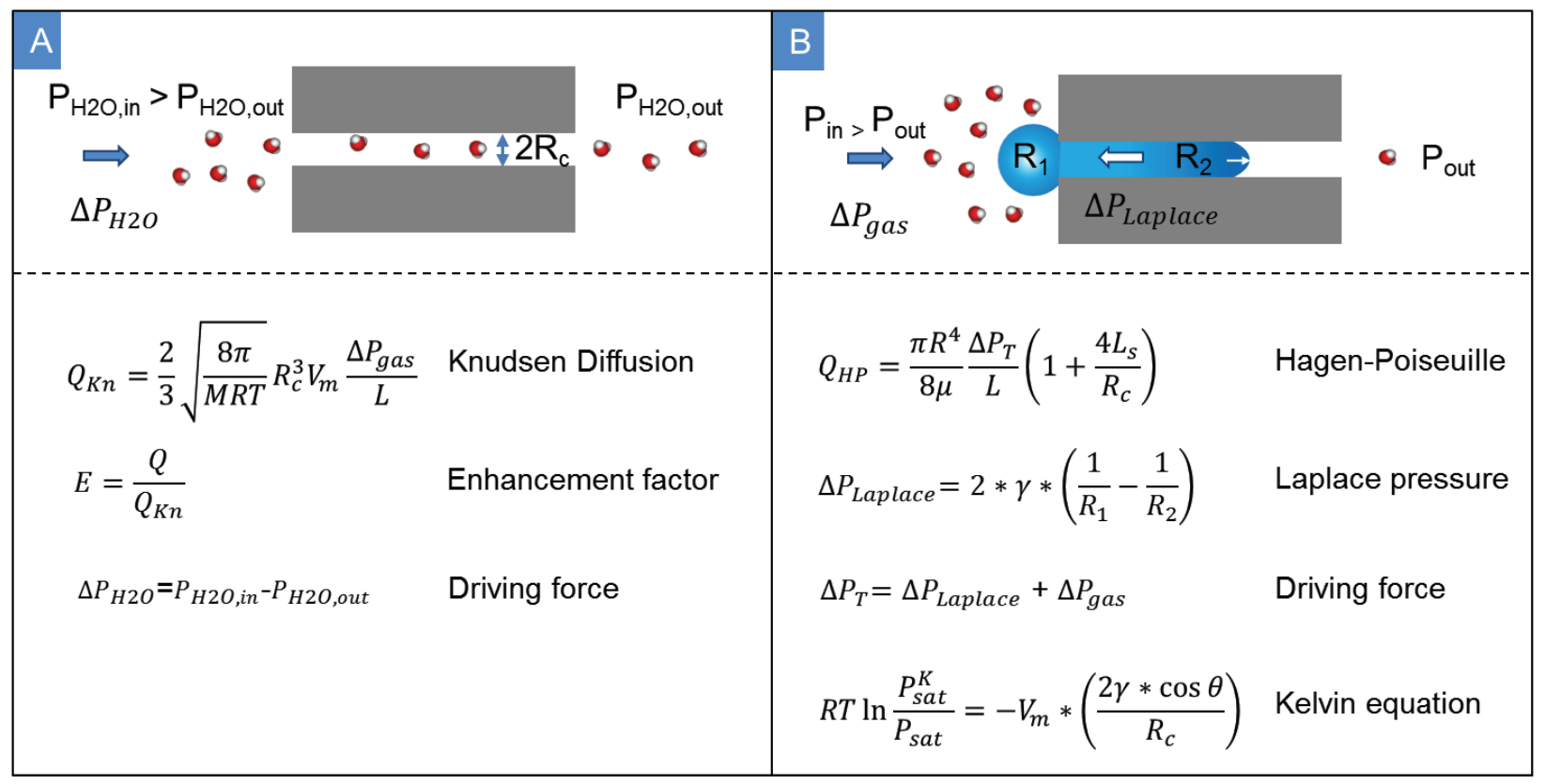

Figure 2. Mechanism of water vapor transport through (A) the endohedral CNT volume and (B) the interstitial space in-between VACNTs. A: Water diffuses through the CNT channels as vapor and with rates that greatly exceed Knudsen predictions [25]. B: According to Kelvin equation, water vapor condenses in the gaps between VACNTs. Formation of an asymmetric liquid meniscus originates a large Laplace pressure difference in the fluid that drives liquid water backwards [41]. $Q_{K n}$ is the volumetric flow rate for Knudsen diffusion; $Q$ the measured volumetric flow rate; $M$ the molecular weigth; $V_{\mathrm{m}}$ the molar volume; $R_{c}$ the capillary radius; $T$ the absolute temperature; $R$ the universal gas constant; $E$ the enhancment factor; $\mu$ is the fluid viscosity; $L$ the pore length; $L_{s}$ the slip length at the pore wall; $R_{1}$ and $R_{2}\left(=R_{c} / \cos \theta\right)$ the radii of curvature of the two menisci; $P^{K}{ }_{\text {sat }}$ and $P_{\text {sat }}$ the saturation pressure of water vapor in equilibrium with a curved liquid meniscus and next to a flat interface, respectively; $\gamma$ the surface tension; $\theta$ the contact angle. 


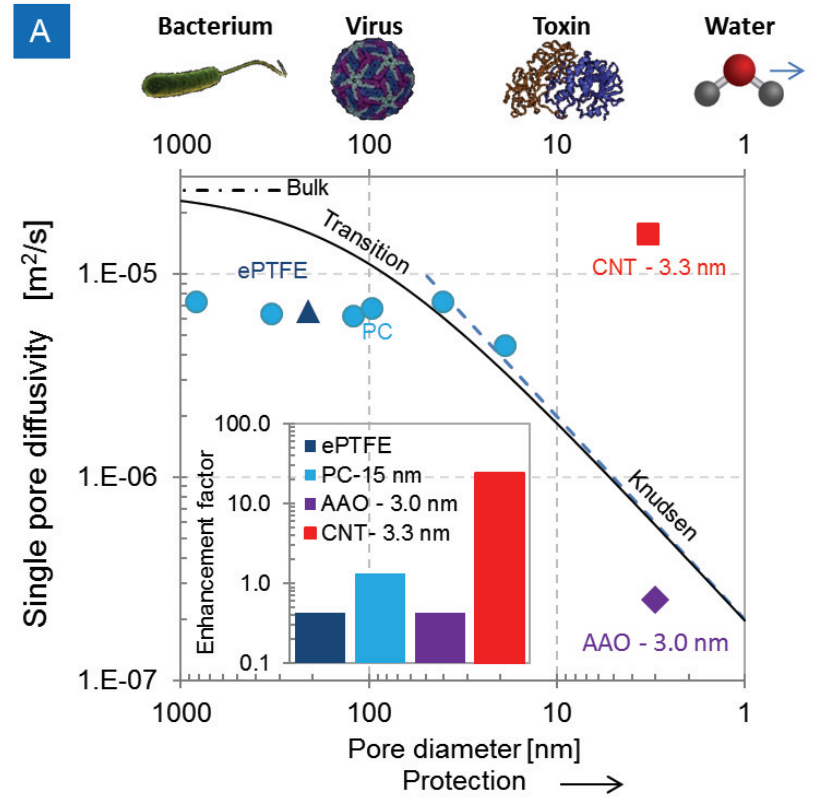

\section{B}

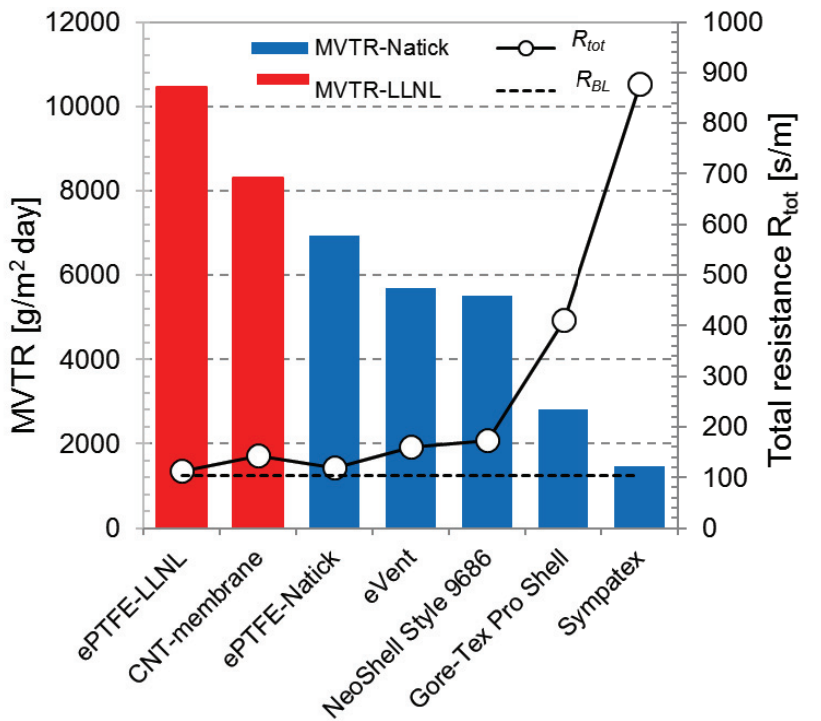

Figure 3. Water vapor transport through VACNT membranes, commercial breathable fabrics, and other porous membranes. A: Single-pore water vapor diffusivity. The pore size dependences predicted by bulk, transition, and Knudsen diffusion equations are represented by the black dotdashed line, solid black line, and the blue dashed line, respectively. Points represent experimental results for several porous membranes. Inset: Enhancement factor defined as ratio of measured to predicted diffusivity. $\mathrm{PC}=$ polycarbonate; $\mathrm{AAO}=$ anodic aluminum oxide. Upper $\mathrm{x}$ axis: Typical dimensions (nm) of biological threats. B: MVTR (bars), corresponding total $\left(R_{t o t}\right.$, circles) and boundary layer resistance ( $R_{B L}$, dashed line) to water vapor transport for CNT membranes and several commercial breathable fabrics based on macroporous films (ePTFE, eVent, NoeShell) or dense polymer laminates (Gore-Tex Pro Shell, Sympatex). Reproduced with permission from [25] - Copyright (C) 2016, John Wiley and Sons. 

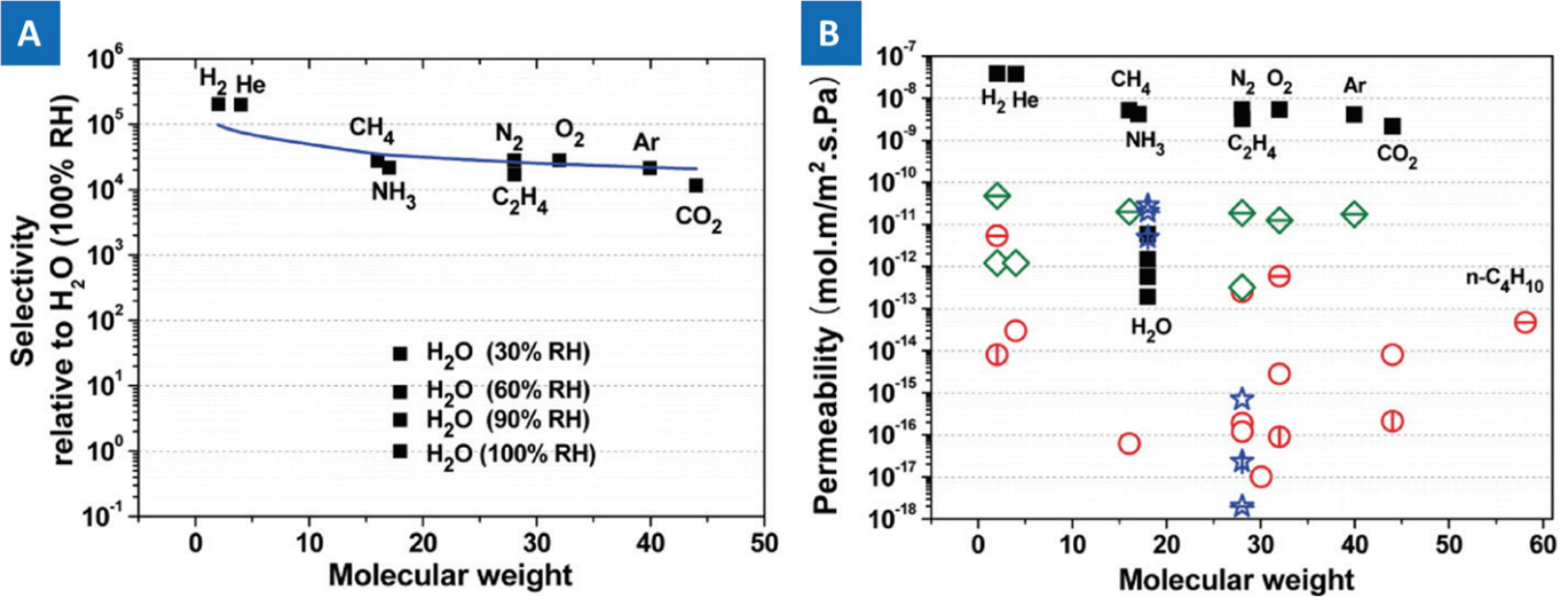

Figure 4. Gas transport through matrixless VACNT membranes. A: Gas selectivity with respect to water vapor when the inlet gas is at $100 \%$ RH. B: Permeability comparison. Data for matrixless VACNT membranes are shown as solid squares, while control data from the literature are represented by the open symbols. Red circles $=$ molecular sieves; green diamonds $=$ carbon nanotubes (inner volume); blue stars $=$ polymeric membranes for water vapor separation . Reproduced with permission from [26] - Copyright (C) 2012, American Chemical Society. 